\title{
Magnetic Profile in Nb/Si Superconducting Multilayers
}

\author{
${ }^{1}$ S.M. Yusuf, ${ }^{1}$ R.M. Osgood III, 'J.S. Jiang, 'C.H. Sowers, 'S.D. Bader, \\ ${ }^{2}$ E.E. Fullerton and ${ }^{1}$ G.P. Felcher
}

'Materials Science Division and Science and Technology Center for Superconductivity
Argonne National Laboratory, Argonne, Illinois 60439

${ }^{2}$ IBM Almaden Research Centre

San Jose, California 95120-6099
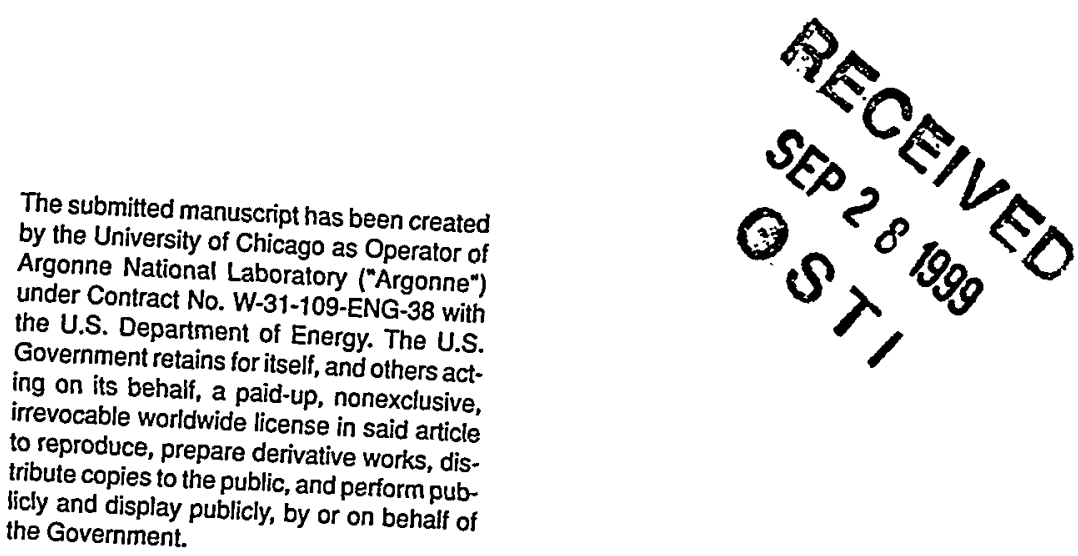

$3^{\text {rd }}$ International Symposium on Metallic Multilayers (MML 98)

Vancouvre, British Columbia, Canada, June 14-19, 1998

Submitted to the Journal of Magnetism and Magnetic Materials .

This work was supported by the U.S. Department of Energy, BES-Material Sciences, under Contract No. W-31-109-ENG-38. 


\section{DISCLAIMER}

This report was prepared as an account of work sponsored by an agency of the United States Government. Neither the United States Government nor any agency thereof, nor any of their employees, make any warranty, express or implied, or assumes any legal liability or responsibility for the accuracy, completeness, or usefulness of any information, apparatus, product, or process disclosed, or represents that its use would not infringe privately owned rights. Reference herein to any specific commercial product, process, or service by trade name, trademark, manufacturer, or otherwise does not necessarily constitute or imply its endorsement, recommendation, or favoring by the United States Government or any agency thereof. The views and opinions of authors expressed herein do not necessarily state or reflect those of the United States Government or any agency thereof. 


\section{DISCLAIMER}

Portions of this document may be illegible in electronic image products. Images are produced from the best available original document. 


\title{
MAGNETIC PROFILE IN Nb/Si SUPERCONDUCTING MULTLAYERS
}

S.M. Yusuf, R. M. Osgood III, J. S. Jiang, C.H. Sowers, S.D. Bader, E.E. Fullerton* and G. P. Felcher .

Argonne National Laboratory, Argonne, II 60439, USA

\begin{abstract}
Polarized neutron reflectivity measurements were conducted on $[\mathrm{Nb}(100 \AA) / \mathrm{Si}(15 \AA)] \mathrm{xN}$ superconducting multilayers with a $T_{c} \sim 7.5 \mathrm{~K}$. Scope of the experiment was to verify the existence of arrays of vortices parallel to the surface above the critical field $\mathrm{H}_{\mathrm{cl}}=400 \mathrm{Oe}$ when a magnetic field was applied parallel to the film plane. Measurements at $1.6 \mathrm{~K}$ and $700 \mathrm{Oe}$ in the zero-field-cooled condition, and at $30 \mathrm{Oe}$ after field cooling, indicated that reflectivity close to the first Bragg reflection of the multilayer was significantly dependent on the neutron spin. This effect was interpreted as due to Josephson type vortices at and around the silicon layers.
\end{abstract}

$\ddagger \quad$ Work supported by US-DOE, BESA-MS contract 31-109-ENG-38.

* Present address: IBM Almaden Research Centre, San Jose CA 95120-6099. 
Artificially layered superconducting-non superconducting structures can be used as model systems of anisotropic superconductors. [1-3]. Most peculiar of these structures is their diamagnetic response to external fields parallel to the layers: above the lower critical field $\mathrm{H}_{\mathrm{cl}}$ diamagnetism exhibits one or more secondary maxima. Possibly the effect has been most studied in $\mathrm{Nb} / \mathrm{Cu}$ multilayers. When these layered superconductors have an overall film thickness larger than the coherence length a series of maxima occurs in the magnetization at specific strengths of the field applied parallel to the surface[4,5]. These maxima have been explained in terms of transitions between specific configurations of the in-plane fluxoids[5]: above $\mathrm{H}_{\mathrm{cl}}$ a first row is formed close to the center of the film; by increasing the field the one-dimensional lattice is compressed until is replaced by two rows of fluxoids with wider spacings, and so on. This description is fundamentally different from that given by Bean for the distribution of magnetization in a layer of type II superconductor, where the fluxoids are simply trapped by impurity centers.

Polarized neutron reflection (PNR) can observe fluxoids in the configuration of fields shown in Fig.1. A neutron beam impinges upon the sample at a typical angle $\theta_{i}$ of the order of one degree. The specularly reflected beam, recorded as a function of the momentum transfer $Q=4 \pi \sin \theta / \lambda$ (where $\lambda$ is the neutron wavelength) contains information on the chemical and magnetic depth profile $z$ of the film. The reflectivities $R_{ \pm}$ for neutrons polarized parallel $(+)$ or opposite $(-)$ to the applied magnetic field are optical transforms of $b(z) N(z) \pm c B(z)$. Here $b(z) N(z)$ is the nuclear scattering amplitude multiplied by the atomic number density and $\mathrm{cB}$ is the spin-dependent magnetic scattering amplitude, expressed in terms of the magnetic induction $B(z)$ averaged over the entire plane at depth $z$ $\left(c=2 \pi m_{n} \mu_{n} / h^{2}=2.3 \times 10^{-10} \AA^{-2} O e^{-1}\right)$. At large values of $Q$, the reflectivities become proportional to 


$$
\mathrm{R}^{ \pm}=\left\{1 / \mathrm{Q}^{+}\right\}[\mathrm{b}(\mathrm{z}) \mathrm{N}(\mathrm{z}) \pm \mathrm{cB}(\mathrm{z})]^{2}
$$

Polarized neutron reflectivity has been used in the past to determine the penetration of external fields in the surface of several superconducting films [6]. The direct comparison of the spin dependent reflectivities is very sensitive to the magnetic scattering amplitude even when this is much lower than the nuclear scattering amplitude (about two orders of magnitudes for $\mathrm{H}_{\mathrm{cl}}=400 \mathrm{Oe}$ ). The effect of fluxoids on the reflectivities depends on their concentration as a function of $z$. For instance, a line of fluxoids close to the center gives rise to a maximal spin dependence of the reflectivity at $Q \sim 2 \pi /(D / 2)(D$ is the total layer thickness); if the fluxoids were pinned at random, their effect would be detected just close to the value of $Q$ corresponding to total reflection.

As seen in Fig.1, a line of fluxoid with spacing $d$ at the center of the layer forms a one-dimensional lattice. This gives rise to an off-specular diffraction line with exit angle $\theta_{f}$ :

$$
(1 / d)=\left(\cos \theta_{f}-\cos \theta_{i}\right) / \lambda
$$

as derived from the conditions of conservation of energy and momentum for the neutrons. However, the geometrical conditions (Eq.2) restrict the occurrence of the line to a limited range of $d$ spacings. Also, the small number of fluxoids means an off-specular diffraction line too weak to be observed directly: instead, we will discuss here just the spin dependence of the reflectivities.

Multilayers of $\mathrm{Nb} / \mathrm{Si}$ were sputtered onto 1 " silicon wafers in 3 mTorr of argon pressure with a substrate temperature of $297 \mathrm{~K}$. The PNR results presented here were obtained on a sample comprising 20 bilayers with $[\mathrm{Nb}(100 \AA) / \mathrm{Si}(15 \AA)]$ nominal composition, protected by a silicon cap $30 \AA$ thick. From $x$-ray reflectivity measurements the thicknesses of the $\mathrm{Nb}$ and $\mathrm{Si}$ layers were found to be $93 \AA$ and $15 \AA$, respectively; the roughness of the $\mathrm{Nb} / \mathrm{Si}$ interface, $7 \pm 1 \AA$ and that of the $\mathrm{Si} / \mathrm{Nb}$ interface $5 \pm 1 \AA$. The superconducting character of these samples was checked by resistivity (with $\mathrm{T}_{\mathrm{c}}=7.5 \mathrm{~K}$ ) and 
by longitudinal magnetization measurements. The zero field cooled (ZFC) magnetization [7] shows two distinct features: below $400 \mathrm{Oe} \mathrm{M} / \mathrm{H}$ is almost constant, indicating full diamagnetic shielding; at higher fields the flux exclusion is reduced and magnetization shows an additional maximum around 900 Oe. The hysteresis loops of Fig. 2 shows that above 400 Oe the superconducting material enters into a mixed regime, and upon reduction of the field a significant amount of flux remains trapped.

The neutron data were taken at the reflectometer POSY I at Argonne. The sample, at a temperature of $1.6 \mathrm{~K}$, was either $\mathrm{ZFC}$ and measured at $\mathrm{H}=700$ Oe or field cooled (FC) at $700 \mathrm{Oe}$ and measured at $\mathrm{H}=30 \mathrm{Oe}$. Fig. 1 shows the spin dependent reflectivities for the $\mathrm{ZFC}$ sample. In the small $\mathrm{Q}$ region, total reflection is observed up to a critical value $\mathrm{Q}_{c}$. Beyond $\mathrm{Q}_{c}$ the reflectivities undergo several rapid oscillations (Kiessig fringes) due to the interference of neutrons reflected from the front and back face of the multilayer. The Bragg peak at yet larger $Q$ is due to the existence of chemical periodicity (108 $\AA$ ) of $\mathrm{Nb} / \mathrm{Si}$. The reflectivity $\mathrm{R}$ was well fitted [7] by the chemical profile obtained from $\mathrm{x}$-rays using as scattering amplitude densities $3.96 \times 10^{-6} \AA^{-2}$ and $2.07 \times 10^{-6} \AA^{-2}$ - the bulk values for $\mathrm{Nb}$ and $\mathrm{Si}$.

The spin dependence can be put in relief by plotting the spin asymmetry, $P=\left(R_{+}\right.$$\left.R_{.}\right) /\left(R_{+}+R_{.}\right)$. $P$ gives the most immediate signature of the magnetic character of the multilayer, being identically zero if the sample is nonmagnetic. It is well known [6] that the Q-region close to total reflection is most sensitive to the penetration in the surface of the external magnetic field. The penetration depth $\lambda_{L}$ for these $\mathrm{Nb} / \mathrm{Si}$ samples has been found[7] to be $1200 \AA$, a value larger by over a factor of two than that of bulk niobium. Fig. 3 (bottom) shows in greater detail the neutron reflectivities over a Q-region covering the first Bragg peak. Fig. 3 (middle) shows the spin asymmetry obtained at 700 Oe in the ZFC state; Fig. 3 (top) shows the spin asymmetry obtained at 30 Oe after FC at $700 \mathrm{Oe}$. The occurrence of a modulation of $\mathrm{P}$ around the Bragg reflection is indicative of an internal 
field distribution with a periodicity commensurate with the $\mathrm{Nb} / \mathrm{Si}$ chemical periodicity. The reversal of its signature indicates that in ZFC the effect is due to fluxoids, in FC to field trapping. The location of finite $\mathrm{P}$ indicates that $\mathrm{Nb} / \mathrm{Si}$ does not have the fluxoid structure proposed for $\mathrm{Nb} / \mathrm{Cu}$ : here the vortices are rather of the Josephson type, centered on each thin Si layers with a pancake-like shape, and spreading out along the layers much farther than across them.

In the context of recent developments this identification does not seem unreasonable. Transport studies have found a coupled 3-D superconducting behavior for such a Nb/Si multilayer system [2]. Recent I-V measurements showed Josephson effects in similar $\mathrm{Nb} / \mathrm{Si}$ multilayers [8]. It has been pointed out [9] that if a Josephson junction is placed in an external field $\mathrm{H}$ and the corresponding Gibbs free energy of a Josephson vortex, $G=4 \Phi_{\mathrm{a}} j_{\mathrm{c}} \lambda_{\mathrm{J}} / \pi \mathrm{c}-\Phi_{\mathrm{o}} \mathrm{H} / 4 \pi$ becomes zero or less, the existence of a Josephson vortex in the interior of the junction is energetically favorable $\left(\Phi_{0}=\right.$ the magnetic flux quantum; $\lambda_{\mathrm{J}}=$ Josephson penetration depth; $\mathrm{j}_{\mathrm{c}}=$ critical current density). Further PNR measurements are needed to elucidate the effect, mapping out the spin-dependent reflectivities as a function of $\mathrm{H}, \mathrm{T}$, the bilayer thickness and eventually its chemical composition. However such program is necessarily quite slow, not only because each single measurement is excruciatingly long, but because the delicate balance of the components makes the exact reproducibility of the samples quite difficult. 


\section{References}

[1] B.Y. Jin and J.B. Ketterson, Adv. Phys. 38 (1989) 189.

[2] J.D. Hettinger, B.R. Washburn, N.B. Remmes, D.G. Steel, K.E. Gray, E.E. Fullerton, and C.H. Sowers, Phys. Rev Lett. 77 (1996) 5280.

[3] W.E. Lawrence and S. Doniach, in Proceedings of the 12th International Conf. on Low Temp. Physics, E. Kanda ed.(Academic Press, Kyoto, 1971) p. 361.

[4] J. Guimpel, L. Civale, F. de la Cruz, J.M. Murduck, and I. K. Schuller, Phys. Rev. B38 (1988) 2342.

[5] S.H. Brongersma, E. Verweij, N.J. Koeman, D.G. de Groot, R. Griessen, and B.I. Ivlev, Phys. Rev. Lett. 71 (1993) 2319.

[6] G.P. Felcher, Physica B 192 (1993) 137.

[7] S.M. Yusuf, E.E. Fullerton, R.M. Osgood III and G.P. Felcher, J. Appl. Phys. 83 (1998) 6801.

[8] I. Vavra, P.Lobotka, J. Derer, S. Gazi, L.R. Wallenberg, V. Holy, J. Kubena, and J.Sobota, J. Low Temp. Phys. 106 (1997) 373.

[9] S.L. Miller, K.R. Biagi, J.R. Clem, and D.K. Finnemore, Phys. Rev. B 31 (1985) 2684. 


\section{FIGURE CAPTIONS}

FIG. 1. Spin dependent reflectivities of a Nb/Si multilayer at 1.6K, in a field of $700 \mathrm{Oe}$ after zero field cooling. Solid dots indicate data for neutron spin parallel to the applied field( $(+)$; open circles for spins antiparallel to the field(-). In the insert, layout of the experiment.

Fig. 2. Minor hysteresis loops of a $[\mathrm{Nb}(100 \AA) / \mathrm{Si}(15 \AA)] \times 30$ sample at $\mathrm{T}=1.6 \mathrm{~K}$, for \pm 700 and \pm 1200 Oe field ranges.

FIG. 3. $1.6 \mathrm{~K}$ reflectivity of a $\mathrm{Nb} / \mathrm{Si}$ multilayer in the region of the first Bragg reflection. Bottom: the spin dependent reflectivity. Center: the spin asymmetry $P=\left(R_{+}-R_{\text {. }}\right.$ )$/\left(R_{+}+R_{-}\right)$measured at $700 \mathrm{Oe}$ after ZFC. Top: spin asymmetry measured at $30 \mathrm{Oe}$ after cooling in a field of 700 Oe. 


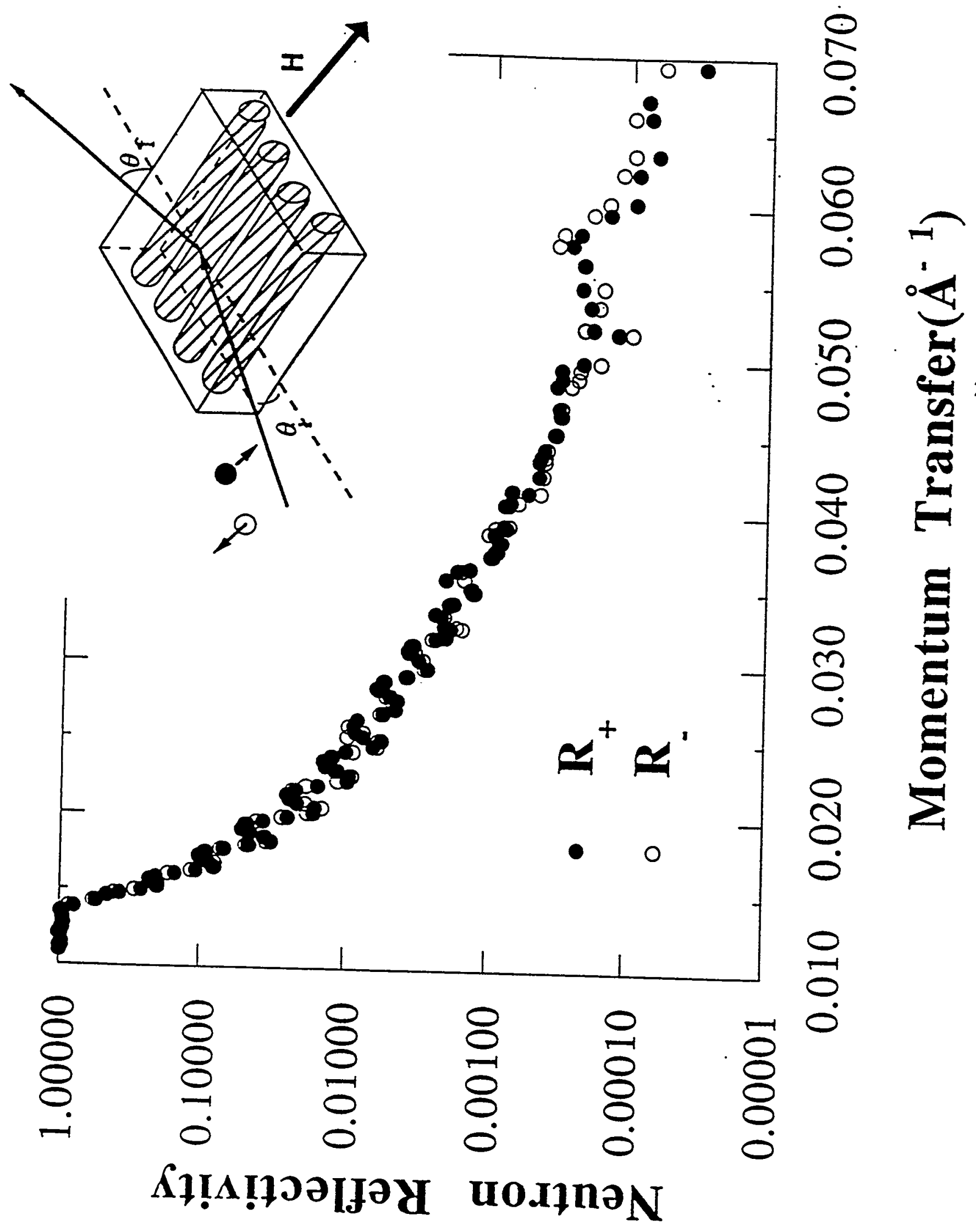



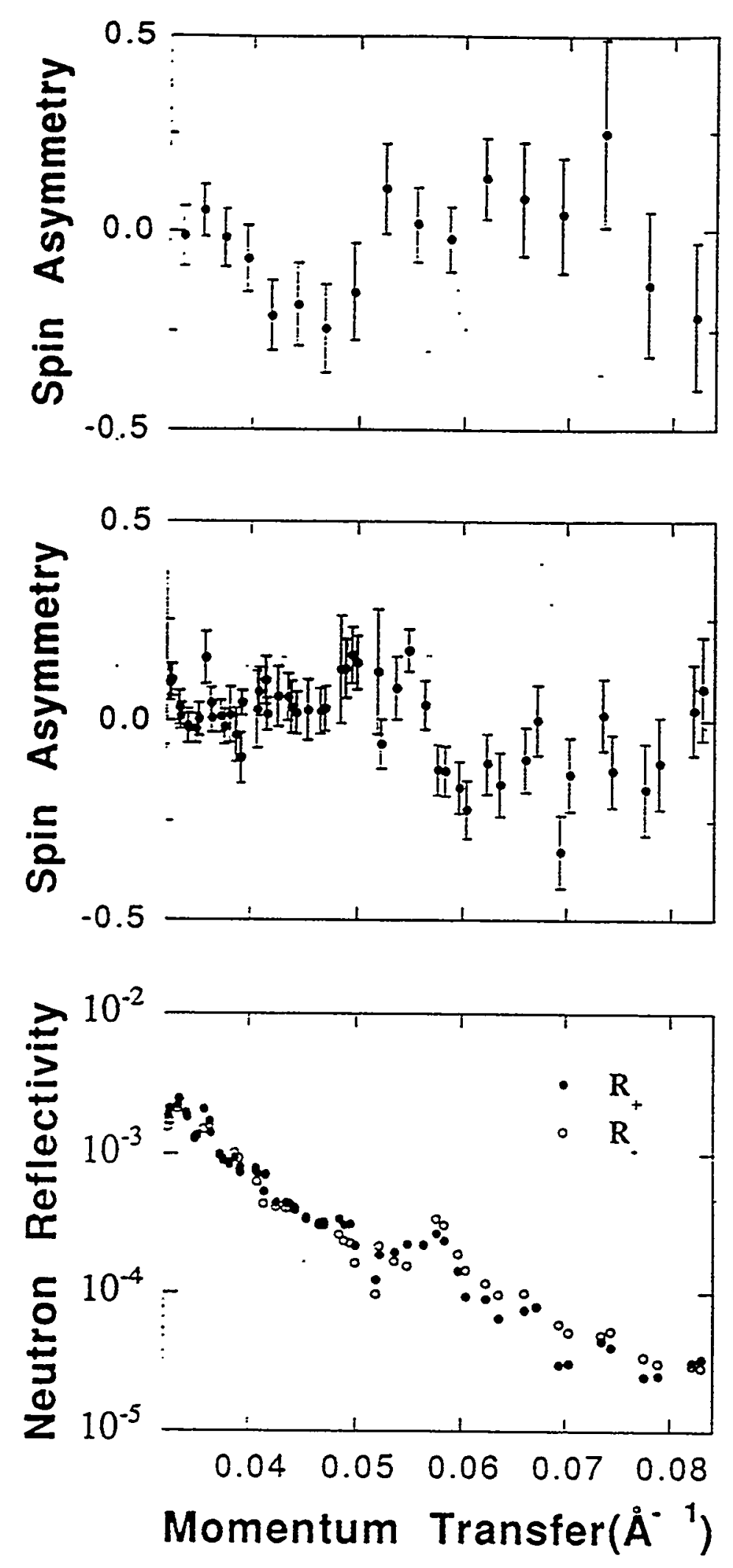